\title{
Indole -3-acetic acid improves drought tolerance of white clover associated with activating auxin- related genes, abscisic acid and jasmonic acid- induced stress responsive transcription factors, and inhibiting senescence genes
}

Youzhi Zhang(Former Corresponding Author)

Sichuan Agricultural University https://orcid.org/0000-0002-7572-4180

Yaping Li

Sichuan Agricultural University

Muhammad Jawad Hassan

Sichuan Agricultural University

Zhou Li

Sichuan Agricultural University

Yan Peng(New Corresponding Author) ( $\square$ pengyanlee@163.com )

Research article

Keywords: drought stress, IAA, phytohormones, auxin-related genes, transcription factor genes, drought resistance genes, senescence genes.

Posted Date: January 20th, 2020

DOI: https://doi.org/10.21203/rs.2.16687/v2

License: (c) (i) This work is licensed under a Creative Commons Attribution 4.0 International License.

Read Full License 


\section{Abstract}

Auxin plays an important role in regulating plant development. To aquire feasible regulation effects and mechanism of IAA on drought tolerance in white clover, relative water content, chlorophyll content, several major phytohormone level, the expression of gene-related to IAA signal perception and synthesis, as well as drought-resistance related transcription factor genes and senescence genes were investigated in white clover plants under $15 \%$ polyethylene glycol-6000 (PEG-6000) after $1 \mu \mathrm{M}$ exogenous IAA pre-treatment and $100 \mu \mathrm{M}$ L-AOPP (inhibitor of IAA synthesis) pre-treatment. Compared to control, drought stress significantly diminished stem dry weight ( $\mathrm{g} / 10$ plants), relative water content (RWC, \%) and total chlorophyll content (Chl, mg/g). Exogenous IAA significantly increased RWC and Chl, however, L-AOPP, drastically decreased stem dry weight (g/10 plants), RWC and Chl compared with direct drought treatment. Besides that, compared with direct drought treatment, exogenous IAA significantly improved ABA content and JA content, up-regulated expression level of auxin-responsing genes (GH3.1, GH3.9, IAA8), drought-resistance related transcription factor genes (bZIP11, DREB2, MYB14, MYB48, WRKY2, WRKY56, WRKY108715) and drought resistance gene (RD22), meanwhile, down-regulated expression level of auxin-responsing genes (GH3.3, GH3.6, IAA27) and leaf senescence genes (SAG101 and SAG102). L-AOPP, contrarily, compared with direct drought treatment, significantly reduced ABA, GA3 and JA content, down-regulated expression level of GH3.1, GH3.9, IAA8, bZIP11, DREB2, MYB14, MYB48, WRKY2, WRKY56, WRKY108715, ERD and RD22, meanwhile, up-regulated SAG101 and SAG102. Collectively, these data suggested a positive role of exogenous IAA in alleviating drought stress damage in white clover.

\section{Introduction}

Drought continuously attracted researchers' concern partly because of its influence in restricting plant growth and distribution worldwide [1, 2, 3]. Adaptability, capability of being or becoming adapted, would give rise to some biochemical and physiological changes to confer plants with improved survival rate in arid environments $[4,5,6,7]$. In essence, these changes are results of expression of direct or indirect related drought responsive genes, including early-response to dehydration (ERD) stress genes and resoponse to dehydration $(R D)$ stress genes which are closely related with plant senescence[8,9] and senescence-associated genes $(S A G)$ which regulate expression of some genes related to chlorophyll degradation and cytoplasm damage[10]. Also, it was reported that transcriptional activation of transcriptional factors (TFs), such as basic region/leucine-zipper motif (bZIP), dehydration responsive element binding (DREB), myeloblastosis $(M Y B)$ and $W R K Y$, could be influenced by drought and regulate plant physiological state.

Futhermore, genes expression are also regulated by hormones, including IAA, CTK, ABA, GA, JA and SA. Among them, IAA, regulating many processes of plant growth and development $[1,4,5,6,11]$, is one of the main naturally occurring phytohormones in plants. Recently, accumulating evidences also indicated possible linkages between auxin and abiotic stresses, even though crosstalks between auxin and other major phytohormones $[12,13,14,15,16,17,18]$. 
Besides that, auxin stimulates RNA synthesis, regulating gene expression response to auxin $[19,20,21$, $22,23]$, which depends on DNA $[24,25]$. In previous studies, auxin response transcription factor $(A R F)$ family could mediate roles of IAA during plant growth [26]. Similarly, TFs could be regulated by one and/or several of phytohormones [27, 28, 29].

White clover (Trifolium repens), one legume plant, is one of important forages for many domesticated animals in the world due to its high yield, good quality and rich nutritional value, and thus is planted in large quantities. However, white clover is susceptible to drought stress and exhibits significant changes in leaf senescence when confronting to dehydration stimulation [30]. However, there are few detailed studies about IAA's effects on dehydration in white clover, from phytohormones to TFs, from IAA responsive genes to senescence genes. In order to explore biochemical and physiological changes of white clover in drought stress and changes caused by IAA addition, we investigated contents of six major phytohormones (including IAA itself) \expression of ARF gene, GH3 family genes (belonging to auxinresponsive gene), IAA repsonse gene, TFs genes, ERD gene and $R D$ gene, $S A G$ genes in white clover under drought stress, with the purpose of provding some possible perspectives of the varied adaptations of white clover in dehydration. And we formulate hypotheses that exogenous IAA improved drouhgt resistance in white clover through several biochemical and physiological changes, ranging from contents of several phytohormones, to expressions of auxin-responsive genes, some TFs genes and senescence genes. These changes confer a better appreance of white clover and reinforce adaptability of it in drought.

\section{Materials And Methods}

\section{Plant material and growth condition}

The seeds of 'Pixie' (Trifolium repens cV.) were purchased from Beijing Mammoth Seed Industry Company in China. The seeds were sterilized with $1 \%(\mathrm{~W} / \mathrm{V})$ sodium hypochlorite solution for $3 \mathrm{~min}$ and then rinsed with sterile water for 3 times, $\sim 0.8 \mathrm{~g}$ of seeds were evenly spread in culture pot $(9 \mathrm{~cm}$ depth, $18 \mathrm{~cm}$ width, $24 \mathrm{~cm}$ length) containing sterilized quartz sand, then placed in the growth chamber $\left(23^{\circ} \mathrm{C} / 16 \mathrm{~h}\right.$ day and $19^{\circ} \mathrm{C} / 8 \mathrm{~h}$ night cycle, irradiance of about $300 \mu$ mol quanta $\cdot \mathrm{m}^{-2} \cdot \mathrm{s}^{-1}, 75 \%$ relative humidity). $25 \mathrm{~mL}$ of deionized water was added to each pot in every day. After germination, instead of deionized water, Hoagland's nutrition solution was used. In order to minimize the environmental effects, the positions of the pots in the culture chamber were also daily replaced.

\section{Experimental design}

When the second leaf of white clover fully unfolded, seedlings were subjected to four sets: (1) Control, Hoagland's nutrition solution was used as previously; (2) PEG-6000 treatment (labeled with D), Hoagland's nutrition solution with 15\% PEG-6000 (W/V, -0.3Mpa); (3) IAA pre-treatment and PEG-6000 treatment (labeled with IAA+D), Hoagland's nutrition solution with $1 \mu \mathrm{M}$ IAA for the first 7-day, and then Hoagland's nutrition solution with 15\% PEG-6000 for the subsequent days; (4) L-AOPP pre-treatment and 
PEG-6000 treatment (labeled with L-AOPP+D), Hoagland's nutrition solution with $100 \mu \mathrm{M}$ L-AOPP for the first 7-day, and then Hoagland's nutrition solution with 15\% PEG-6000 for the last days. All plants were simultaneously treated with PEG-6000. Each treatment had 4 biological replicates. Samples were collected on $0 \mathrm{~d}, 7 \mathrm{~d}$ and $14 \mathrm{~d}$ after adding PEG-6000.

\section{Physiological measurements}

\section{Relative water content (RWC), stem dry weight and Total Chlorophyll content (Chl)}

For RWC, approximately $0.2 \mathrm{~g}$ of leaves (FW) were wrapped with clean gauze and then dipped into distilled water. After 24 hours at $4{ }^{\circ} \mathrm{C}$, weighed them as saturated weight (SW). Samples were placed in kraft paper bags, kept in an oven at $105^{\circ} \mathrm{C}$ for $30 \mathrm{~min}$, then at $75^{\circ} \mathrm{C}$ for $48 \mathrm{~h}$, and weigh them as dry weight (DW). RWC was calculated through formula [31]: RWC(\%)=(FW-DW)/( SW-DW $) \times 100 \%$ D

For total chlorophyll content, approximately $0.1 \mathrm{~g}$ of leaves (FW) were extracted with $10 \mathrm{~mL}$ of mixed solution $(80 \%$ acetone $/ 95 \%$ methanol, $1: 1 \otimes \mathrm{V} / \mathrm{V})$ in glass tubes in dark till leaves became colorless. The extract solution was instantly assayed spectrophotometrically. Specific absorption of $\mathrm{Chl}$ a and $\mathrm{Chl} b$ was recorded at $645 \mathrm{~nm}$ and $663 \mathrm{~nm}$, respectively. Total chlorophyll content was calculated according to the following formula [32]:

$\operatorname{Chl}(\mathrm{a}+\mathrm{b})(\mathrm{mg} / \mathrm{g})=\left(20.2 \times \mathrm{OD}_{645}+8.02 \times \mathrm{OD}_{663}\right) /(\mathrm{DW} \times 1000) \square$

\section{Quantification of IAA, ABA, CTK (iPAs and ZRs), GA3, JA and SA}

HPLC-ESI-MS method [33] with minor modification was used to quantify IAA, ABA, CTK (iPAs and ZRs), GA3, JA and SA. Leaves of white clover were weighted $(\sim 0.2 \mathrm{~g})$ and transferred to a $5 \mathrm{ml} \mathrm{screw-cap}$ tube with one steel ball whose diameter was less than $5 \mathrm{~mm}$. Kept tubes in liquid nitrogen for 10 min to frezze plant samples and ground them into powder through a Plant Tissue Breaker. Add $200 \mu \mathrm{l}$ of the working solution of internal standards [33] to each tube. Add $2 \mathrm{ml}$ extraction solvent [33]. Shake tubes at a speed of $100 \mathrm{rpm}$ for $30 \mathrm{~min}$ at $4{ }^{\circ} \mathrm{C}$. Add 2 dichloromethane to each tube and shake for $30 \mathrm{~min}$ in a $4{ }^{\circ} \mathrm{C} \mathrm{room}$. Centrifuge at a speed of $12,000 \mathrm{rpm}$ for $5 \mathrm{~min}$ at $4{ }^{\circ} \mathrm{C}$. Transfer $\sim 1.8 \mathrm{ml}$ of the solvent from the lower phase into a screw-cap vial and concentrate it by using a nitrogen evaporator. The samples were redissolved in $0.2 \mathrm{ml}$ methanol. Inject $20 \mu \mathrm{l}$ of the last methanol solution into the C18 (reverse-phase) HPLC column for analysis. The HPLC-ESI-MS (HPLC system, LC-10AD series, Shimadzu, Japan; ESI-MS system, ABI 4000 QTRAP) conditions and settings were used. Applied Biosystems Analyst software version 1.5.1 was used to control MS system and to perform data analysis and data management.

\section{Expression analysis of genes related to IAA}

The expression of GH3.1, GH3.3, GH3.5, GH3.6, GH3.9, IAA8, IAA27 and ARF in Trifolium repens leaves were evaluated through real-time quantitative PCR (qRT-PCR). Total RNAs extraction from Trifolium repens leaves were performed through Plant RNA Kits (TianGen Biochemical Technology Co., Ltd) and their reverse transcriptions through iScriptTM cDNA Synthesis Kit (Bio-Rad company). cDNAs were used 
as templates to do qRT-PCR $T$ The $10 \mu \mathrm{L}$ reaction system consisted of template CDNA $1 \mu \mathrm{L}$, upstream primer $(10 \mu \mathrm{M}) 0.5 \mu \mathrm{L}$, downstream primer $(10 \mu \mathrm{M}) 0.5 \mu \mathrm{L}$, SYBR Green Super Mix $5 \mu \mathrm{L}$ and $\mathrm{ddH}_{2} \mathrm{O} 3 \mu \mathrm{L}$. The reaction procedures were: pre-denaturation $3 \mathrm{~min}$ at $95^{\circ} \mathrm{C}$, denaturation $10 \mathrm{~s}$ at $95^{\circ} \mathrm{C}$, annealing 15 s at $59.3{ }^{\circ} \mathrm{C}(G H 3.1), 61^{\circ} \mathrm{C}(G H 3.5), 60^{\circ} \mathrm{C}\left(G H 3.3, G H 3.6, G H 3.9\right.$, IAA8, IAA27) and $58.9^{\circ} \mathrm{C}(A R F)$, extension $20 \mathrm{~s}$ at $72{ }^{\circ} \mathrm{C}, 30$ cycles, final extension $10 \mathrm{~min}$ at $72{ }^{\circ} \mathrm{C}$. GAPDH was used as reference gene to correct number of template copy, then the relative expression of each gene was calculated according to the formula of $2^{-\Delta \Delta C t}$ [34]. Primer sequences of the genes related to IAA and their corresponding GeneBank accession numbers were listed in Table 1 in Appendixes.

\section{Expression of drought-induced transcription factor (TF) genes, response to drought (ERD/RD22) and senescence-associated genes(SAG101/SAG102)}

cDNAs were used as templates in qRT-PCR. The $10 \mu \mathrm{L}$ reaction system consisted of template cDNA $1 \mu \mathrm{L}$, upstream primer $(10 \mu \mathrm{M}) 0.5 \mu \mathrm{L}$, downstream primer $(10 \mu \mathrm{M}) 0.5 \mu \mathrm{L}$, SYBR Green Super Mix $5 \mu \mathrm{L}, \mathrm{ddH}_{2} \mathrm{O} 3$ $\mu \mathrm{L}$. The reaction procedures were: pre-denaturation $3 \mathrm{~min}$ at $95^{\circ} \mathrm{C}$, denaturation $10 \mathrm{~s}$ at $95^{\circ} \mathrm{C}$, annealing

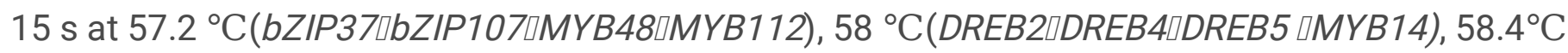
(WRKY108715), $61^{\circ} \mathrm{C}(W R K Y 2 \square W R K Y 56 \square b Z I P 11), 56.4{ }^{\circ} \mathrm{C}(R D 22)$ and $59.5^{\circ} \mathrm{C}(E R D), 55.5^{\circ} \mathrm{C}$ (SAG101/SAG102), extension 20s at $72{ }^{\circ} \mathrm{C}, 30$ cycles, final extension $10 \mathrm{~min}$ at $72{ }^{\circ} \mathrm{C}$. GAPDH was used as reference gene to correct number of template copy and then the relative expression of each gene was calculated according to the formula of $2^{-\Delta \Delta \mathrm{Ct}}$ [34]. Primer sequences of drought-induced transcription factors and drought-induced genes and their corresponding GeneBank accession numbers are listed in Table 2 in Appendixes.

\section{Statistics and mapping}

In this paper, Origin 8.5.1 was used to generate the histogram, SPSS 19.0 to analysis of variance (ANOVA) at 0.05 probability level. Data were transformed to meet normality and homogeneity of variance. Fisher's LSD was used to determine differences among sets.

\section{Results}

\section{Effects of exogenous IAA on drought tolerance of white clover}

Morphological appearance of white clover plants in all experiment sets were shown as Figre 1A. IAA pretreatment had an obvious facilitation to white clover under normal condition, followed by a significant higher stem dry weight as compared to other treatments (Figure 1B). However, L-AOPP (inhibitor of auxin

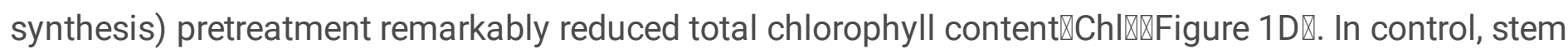
dry weight increased (Figure 1B) by days, RWC and Chl remained stable throughout the experiment (Figure1C-1D). When drought stress went on, stem dry weight, RWC and Chl content gradually decreased in white clover in all sets (Figure1C-1D). However, stem dry weight, RWC and Chl were significantly higher in IAA pretreatment than those without IAA pretreatment. Furthermore, L-AOPP significantly diminished them when compared to drought sets (Figure 1B-1D). 


\section{Content of ABA, CTK (iPAs and ZRs), GA3, JA and SA}

On 0 day, IAA reduced CTK content by $90.2 \%$ and improved GA3 and JA content by $45.2 \%$ and $18.4 \%$ (Figure 2B-2D), respectively. And, it had no influence on ABA and SA content (Figure 2A-2E). However, LAOPP increased CTK content by $61.4 \%$ and SA content by $130 \%$ (Figure $2 B, 2 E$ ), decreased GA3 and JA content by $28.8 \%$ and $13.8 \%$ (Figure $2 \mathrm{C}, 2 \mathrm{D}$ ), respectively. And it had no impact on ABA content (Figure 2A). In PEG-6000 treatment, ABA and JA content gradually got rose, accompanied by the gradual decline in CTK, GA3 and SA content during experiment period. On 7 day and 14 day, ABA, GA3 and JA contents were significantly higher in IAA set than those without IAA pretreatment (Figure 2A, 2C, 2D), while SA content was significantly lower in IAA set than it in others. L-AOPP nearly had the opposite appearances when compared with IAA (Figure 2A, 2C, 2D).

\section{Endogenous IAA content and relative expression of auxin response genes}

IAA set showed a significant increase in endogenous IAA content while L-AOPP set exhibited a significant decrease of endogenous IAA content in white clover under normal condition. The content of endogenous IAA remained at a stable level in control but decreased much in PEG-6000 treatment from start to end (Figure 3A). However, compared with PEG-6000 treatment, exogenous IAA pretreatment maintained a considerably higher endogenous IAA content in white clover exposed to stress, inversely, the L-AOPP pretreatment showed a remarkable decreased trendancy in endogenous IAA content (Figure 3A).

Under normal condition, exogenous IAA improved the expression of $A R F$ (auxin response factors), GH3.9 and $I A A 8$, lowered the expression of GH3.5 and IAA27, and had no effects on the expression of GH3.1, GH3.3 and GH3.6. L-AOPP mainly restrained the expression of GH3.1 and GH3.3 and had no prominent influence on other genes. PEG-6000 inhibited the expression of $A R F$, up-regulated the expression of other genes. Under drought stress, significantly higher expression level of $A R F, G H 3.1, G H 3.5, G H 3.9$ and IAA8 and lower expression level of $G H 3.3, G H 3.6$ and IAA27 were detected in IAA set compared to IAA-absence set. However, L-AOPP down-regulated all genes expression except GH3.5 (Figure 3B-3J) .

\section{Relative expression of transcription factor (TF) genes responsed to drought stress in white clover}

In this experiment, we chose 3 genes in $b Z I P, D R E B, M Y B$ and WRKY transcription factor family, respectively. Under no stress, IAA significantly up-regulated expression of $b Z I P 107, M Y B 48, W R K Y 2$ and WRKY56, down-regulated expression of $D R E B 5$ and $M Y B 48$, and had no remarkable influence on expression of $b Z I P 11, b Z I P 37$, DREB2, DREB4, MYB14 and WRKY108715. L-AOPP significantly downregulated $b Z I P 107$ and up-regulated $D R E B 5$. All concerned transcription factor genes were induced by PEG-6000 except that expression of $b Z I P 107$ was depressed in present experiment. Under drought stress, addition of IAA further up-regulated the expression level of all the transcription factor genes, L-AOPP lowered the expression of these genes, exception for $b Z I P 37$ and $b Z I P 107$ compared with PEG-6000 set (Figure 4B, 4C).

\section{Expression of drought-response genes and senescence-associated genes}


Under no stress, exogenous IAA showed a conspicuous inhibition to the relative expression of $E R D$. However, L-AOPP significantly induced expression of $R D 22$ gene. Drought enhanced expression of $E R D$ and $R D 22$ in large. IAA also improved expression of ERD and RD22 and L-AOPP lowered their expressions (Figure 5). In addition, drought stress extremely activated expression of SAG101 and SAG102, L-AOPP upregulated expression of $S A G 101$, while IAA displayed a prominent suppression to expression of SAG101 and SAG102 in white clover.

\section{Discussion}

\section{Improved growth and physioloies in white clover}

Under drought stress, our results showed that exogenous $1 \mu \mathrm{M}$ IAA pretreatment on root mitigated plant wilt whereas L-AOPP worsened it (Fig. 1A). Meanwhile, IAA pretreatment significantly improved stem dry weight, relative water content and total chlorophyll content in leaves, however, L-AOPP decreased all of them (Fig. 1B-1D). Studies have shown that IAA is closely related to drought tolerance in plants, and wild type Arabidopsis plants pre-treated with IAA exhibited enhanced drought resistance [35]. Under water deficiency, IAA effectively maintained relative water content and enhanced photosynthetic efficiency and growth of barley. Application of IAA could alleviate the adverse effects brought by dehydration and succeed in enhancing barley growth [36]. Apparently, IAA made morphological and physiological state of white clover in IAA+D set much better than in PEG-6000 set (Figure1). In contrast to appearance of plants in L-AOPP+D, it could be concluded that IAA pretreatment had a positive effect in improving drought tolerance in white clover.

\section{Content Variations of Endogenous Phytohormones}

It was showed that drought stress induced an increase in ABA and JA content, but a decrease in CTK, GA3 and SA content (Figure 2A-2E). IAA significantly increased ABA, GA3 and JA content (Figure 3A, 3C, $3 D)$. These results implied that IAA played an important role in synthesizing or accumulating ABA, GA3 and JA in white clover.

Transcriptome data revealed that increase of $A B A$ content induced and activated the expression of a large number of drought resistant genes [37]. $A B A$ regulated downstream response of $R D 29 B$ (dehydration stress gene) by regulating $b Z I P$ gene [38]. In our studies, we also found that there was a consistent correlation between content of $\mathrm{ABA}$ and expression of $R D 22$ under stress (Figure 3A, Figure $5 \mathrm{~B})$, suggesting that $\mathrm{ABA}$ also probably regulated expression of $R D 22$ gene in white clover.

In Arabidopsis and rice, the accumulation of ABA regulated the polar transport of auxin $[39,40]$. It has been found that the interaction between IAA and ABA promoted the development of lateral roots in plants, and this pattern of root growth regulation was important for plants to respond to severe drought stress [41]. Besides, exogenous ABA enhanced the recovery of photosynthetic rate in upland rice under PEG stress [42]. Based on these experimental results and combined with Figure 1 and Figure 3 A, we also could speculate that an increase in ABA content enhanced drought resistance through multiple ways, 
such as improved RD22 gene expression, more content of total chlorophyll and more stem dry weight. However, L-AOPP had opposite effects on them, further confirming that these changes were caused by IAA.

As far as IAA and GAs were concerned, some researchers proved that normal level of bioactive GA1 required normal level of IAA in elongating pea stems [43] and IAA promoted GA1 synthesis [44]. It was found that GA induced the formation of porosity [45]. Here, our results showed that content of exgenous IAA significantly increased the content of GA3 (Figure 3C), and IAA content and GA3 content had consistency in change. And this consistency probably was a result of IAA's activation in enzemes ralated to GA3 synthesis, like IAA's promotion on GA1 synthesis [44].

Transgenic creeping bentgrass over-expressing prenyltransferase had a higher endogenous CTK content and improved photosynthesis and water use efficiency and further enhanced drought resistance [46]. In the interaction between IAA and CTK, it was found that IAA down-regulated biosynthesis level of CTK [47]. Our results showed that the increase in endogenous IAA content significantly decreased content of CTK under water sufficiency, but had no significant effect on the level of CTK under drought stress. It was found that CTK inhibited auxin transport protein PIN and reduced the accumulation of IAA, but inhibited lateral root growth [48]. However, the application of CTK resulted in a rapid increase in IAA in young parts [49]. According to these results, interaction between CTK and IAA possibly could be complicated and dependent on different tissues.

JA content increased rapidly under drought stress, and a strong interaction between JA and ABA signaling pathway was observed [50]. Our results indicated that exogenous IAA also increased content of JA (Figure 3D). Some studies have shown that JA is in the upstream of ABA biosynthesis, and the accumulation of JA at early stage led to accumulation of jasmonic acid isoleucine, which is one necessary condition for $A B A$ synthesis under drought stress [51]. Like these results, improved content of JA could spur content of ABA to an improved level.

Exogenous application of SA could improve photosynthetic activity, leaf water content and membrane permeability, thus enhanced tolerance of tomato to drought [52]. Our studies found that content of SA was decreased by drought stress but not regularly influenced by exogenous IAA. Additionally, AlonsoRamírez A found that GA positively regulated SA and exogenous application of GA could increase content of SA and alleviated environmental stress [53]. Here, GA3 probably also alleviated drought stress through some certain regulatory ways except its influence on variation of SA content.

Taken together, we speculated that the altered phytohormones tend to reach to a new homeostasis after application of exogenous IAA under drought stress. These variations of major phytohormones could contribute to drought resistance for plants through certain signal transduction and gene regulation pathways.

\section{Changes of expression of genes responsing to IAA and TF genes}


Transcriptome data showed that rice $A U X / I A A$ genes were induced by exogenous IAA and drought [54]. AUX/IAA1 in Sorghum was also up-regulated by drought [55]. IAA8 and IAA27 gene relative expression got a homeostasis during all time when water was sufficient. Drought stress enorrmously prompted their expressions on $7 \mathrm{~d}$ and $14 \mathrm{~d}$. We found that exogenous IAA up-regulated expression of IAA8 and down regulated expression of IAA27. In zinnia, transcript level of IAAB was particularly induced by auxin and was expressed in plant vascular development [56]. Tomato transgenic plants with under-expression of $S /$ IAA27 gene showed multiple phenotypes interrelated to vegetative growth. Silencing of it resulted in higher auxin sensitivity, with change of root development and diminished Chl content in leaves [57]. Here, down-regulation of IAA27 also probably had multiple effects on growth and root development in white clover.

High content $A R F$ gene expression nearly corresponded to high content of endogenous IAA, meaning that ARF genes expression were largely modulated by endogenous IAA. One study also found that auxin treatment could affect transcript abundance of seveal OSARF genes, and these ARF genes might play crucial roles in varied metabolic pathways and some cellular processes in rice [58]. At present, there are few reports on the functional verification of ARF gene in other plants. It is necessary to further study the specific role of $A R F$ gene in plants responsing to IAA and drought stress.

GH3 family genes were also involved in plant responsing to biotic and abiotic stresses. Our studies showed that expressions of GH3.1, GH3.3, GH3.6 and GH3.9 were induced by drought stress (Figure 3C, $3 \mathrm{D}, 3 \mathrm{~F}$ and $3 \mathrm{G}$ ), denoting that these $\mathrm{GH}$ family genes could respond to drought stress. Besides, exogenous IAA also prompted expressions of GH3.1 and GH3.9 genes (Figure 3C and 3G), indicating that these two genes may have relation to endogenous IAA content. Arabidopsis thaliana seedlings pretreated with IAA showed strong drought tolerance, and other studies showed that exogenous IAA regulated a variety of gene expression related to stress [59]. It was found that decreased endogenous IAA content in rice mutants accompanied with deficiency in carotenoid and transgenic plants overexpressing OsGH3.2 showed the sensitivity to drought [11]. Activation of OsGH3.13 enhanced drought resistance in Rice [60]. Moreover, exogenous IAA activated responsive gene GH3.9 and resulted in the strong drought resistance in plant [61]. These results indicated that exogenous IAA could enhance drought resistance in white clover and $G H 3.1$ and $G H 3.9$ gene was involved in drought tolerance indeed.

Transcription factors (TFs) play important regulatory roles in growth, development, morphogenesis and response to external environments. At present, hundreds of transcription factors regulating plant resistance to drought, low temperature, high salt and disease have been found in higher plants. They were divided into $b Z I P, D R E B, M Y B$ and WRKY family groups etc, according to differences of DNA binding domains. Due to their abilities to regulate a number of genes associated with stress, TFs activations by special phytohormone are important to improve stress resistance of plants.

For bZIPS, only a small part of them were identified to play roles in plant growth and development, abiotic stress and hormone signal transduction, however their potential molecular mechanisms are still unknown, and need further exploration [62]. Previous study has shown that OsbZIP23 in maize is 
involved in ABA signalings and actively regulates drought and salt stress [63]. Other researchers found that bZIP11 in Arabidopsis interacted with one adapter proteins via an amino-terminal activation domain to recruit histone acetylation system to specific auxin-responsive genes [29]. bZIP37 expressed highly in the salt-stressed plant, which might effectively activated downstream of ABA-inducible gene expression [64]. We found that expression of bZIP11 was also induced by exogenous IAA (Figure 4A), and that of $b Z I P 37$ was induced by PEG-6000 stress (Figure 4B). So, we speculated that $b Z I P 11$ gene responded to IAA and $b Z I P 37$ responded to drought stimulus.

$D R E B s$ (dehydration-responsive element-binding proteins) play important roles in plant response to drought stress and were found to be activated in ways dependent on ABA or not [65]. It was showed that exogenous IAA positively enhanced expression of DREB2 and DREB4, and L-AOPP negatively regulated expression of DREB2 and DREB4 (Figure 4D, 4E) in our studies. Other study has shown that DREBs regulate expression of many downstream genes of drought resistance and over-expression of DREB gene can enhance drought resistance in plants [66]. Our results showed that the improved drought resistance of white clover by exogenous IAA could be associated with the role of DREB2 and DREB4 expression.

MYBs also are important in regulating plant growth, development, metabolism and stress response, and almost all eukaryotes have $M Y B$ transcription factors. The response mechanisms of $M Y B s$ in stress environment are not very clear. Our studies found that both exogenous IAA and drought stress positively regulated expression of MYB14 and MYB48 and L-AOPP decreased their expression (Figure 4G, 4H). Xiong found that over-expression of $M Y B 48-1$ promoted biosynthesis of $A B A$ and improved drought resistance of transgenic rice [67]. AtMYB60 regulated stomatal movement and promoted Arabidopsis thaliana to respond to drought stress [27]. Different MYBs showed varied functions in progress of responding to drought and improved drought resistance directly or indirectly.

At present, the research progress of WRKY transcription factors in abiotic stress has been gradually developed. It was found that cold, heat, salt, drought and hormone induced WRKY gene expression quickly. WRKYs also played important roles in plant drought stress and regulated plant response to abiotic stress through interaction with hormones and protein kinases [68], However the molecular mechanism of its regulation were still limited. It was found that WRKYs were involved in plant stress regulatory networks and WRKY proteins expressions were induced by drought stress [69]. WRKY transcription factor $A B O 3$ induced expression of drought resistance genes, such as RD29A and COR47, and positively regulated drought resistance [70]. In terms of WRKY2, WRKY56, and WRKY108715 genes, we found that drought also induced their expressions. Moreover, exogenous IAA also significantly upregulated expression levels of $W R K Y$ family genes, comparing with direct drought treatment. It seemed that these WRKYs played considerably important role in white clover response to drought and IAA improved capability of this response.

\section{Expression of stress gene and senescence -associated gene}

In term of $E R D$ and $R D 22$ genes, our results suggested that drought up-regulated expression levels of them. Furthermore, exogenous IAA also prompted their expression levels. L-AOPP decreased their 
expression levels. Several studies also showed that high expression level of $E R D$ and $R D 22$ subserved plant resistance to drought $[71,72]$. ERD11 and ERD13 genes could encode some polypeptides which were homologous to glutathione S-transferases in tobacco and maize. Besides, expressions of ERD11 and ERD13 genes were induced by dehydration, but not influenced by GA, ABA, 6-BA and 2,4-D [73]. Other studies also found that $R D 22$ gene was double improved by both ABA and MYB proteins [74].

As far as SAG101 and SAG102 genes were concerned, our studies found that drought extremely improved their expression levels, IAA significantly decreased expression levels of them and L-AOPP enhanced expression levels of them. SAG101 in Arabidopsis encoded an Acyl Hydrolase involved in leaf senescence [75]. It was found that exogenous IAA inhibited transcription level of SAG12 [76] and retarded senescence of leaves. The plant with over-expression of YUCCA6 gene improved content of endogenous IAA and hindered senescence of plant through down-regulated expression of SAG12 [77]. Similarily, the depressed expression of SAG101 and SAG102 by IAA could play a part in delaying senescence resulted from drought stress in white clover.

\section{Conclusion}

Under normal condition, endogenous IAA content of leaves in IAA+D was the highest (Figure 3A). Under PEG-6000 treatment, IAA pretreament on roots of white clover improved endogenous IAA level while LAOPP pretreatment decreased IAA content of leaves (Figure 3A). As an important plant hormone, IAA induced changes in other major phytohormones in white clover, altering expressions of multiple genes which included auxin response, transcriptional factors, drought resistance and leaf senescence. At last, morphological appearance and physiological adaptations demonstrated variations.

This study demonstrated a positively protective role of exogenous IAA on drought resistance in white clover.

\section{Declarations}

Author information:

Corresponding Author

*E-mail: pengyanlee@163.com. Phone number: +86-028-86291010. Fax: +86-028-86291010.

ORCID

Yan Peng: 0000-0001-7776-877X

Author Contributions:

Youzhi Zhang, Yaping Li, Zhou Li and Yan Peng conceived the research and designed the experiments; Yaping Li performed the experiments; Youzhi Zhang analyzed the data; Youzhi Zhang worte the 
manuscript; Yaping Li, Zhou Li, Yan Peng and Muhammad Jawad Hassan discussed the results and reviewed the manuscript. All authors have given approval to the final version of the manuscript.

co: These authors contributed equally.

Notes: The authors declare no competing financial interest.

Acknowledgements: This research was supported by Grant No. 2018H H0067 from the International Cooperation Project of Sichuan Province and by Provincial Industry Independent Innovation Ability Project in Jilin Province: Construction of Independent Innovation Ability of Applied Eco-engineering Laboratory in Jilin Province by Grant No.2018c002.

\section{References}

1. Harb, A., Krishnan, A., Ambavaram, M. M. R., \& Pereira, A.. Molecular and physiological analysis of drought stress in arabidopsis reveals early responses leading to acclimation in plant growth. Plant Physiology. 2010;154(3):1254-1271.

2. Shi H, Ye T, Chan Z. Comparative proteomic responses of two bermudagrass (Cynodon dactylon (L). Pers.) varieties contrasting in drought stress resistance. Plant Physiology and Biochemistry. 2014; 82:218-228.

3. Li Z, Zhang Y, Zhang X, Merewitz E, Peng Y, Ma X, Huang L, Yan Y. Metabolic Pathways Regulated by Chitosan Contributing to Drought Resistance in White Clover. Journal of proteome of research. 2017; 16:3039-3052.

4. Sean R. Cutler, Pedro L. Rodriguez, Ruth R. Finkelstein. Abscisic Acid: Emergence of a Core Signaling Network. Annual Review of Plant Biology. 2010; 61:651-679.

5. Takashi Hirayama, Kazuo Shinozaki. Research on plant abiotic stress responses in the post-genome era: past, present and future. The plant Journal. 2010; 61(6): 1041-1052.

6. Qin F., Shinozaki K., Yamaguchi-Shinozaki K (). Achievements and challenges in understanding plant abiotic stress responses and tolerance. Plant Cell Physiol. 2011;52:1569-1582.

7. Julia Krasensky, Claudia Jonak. Drought, salt, and temperature stress-induced metabolic rearrangements and regulatory networks. Journal of Experimental Botany. 2012; 63(4): 1593-1608.

8. Kiyosue T, Yamaguchi-Shinozaki K, Shinozaki K.Cloning of cDNAs for genes that are early-responsive to dehydration stress (ERDs) in Arabidopsis thaliana L.: identification of three ERDs as HSP cognate genes. Plant Molecular Biology. 1994;25(5): 791-798.

9. Rahma Jardak Jamoussi, Mohamed Malek Elabbassi. Physiological responses of transgenic tobacco plants expressing the dehydration responsive RD22 gene of Vitis vinifera to salt stress. Turkish Journal of Botany. 2014;38: 268-280.

10. Gan S, Amasino R M. Making sense of senescence (molecular genetic regulation and manipulation of leaf senescence). Plant physiology. 1997; 113(2): 313. 
11. Du H, Wu N, Fu J. A GH3 family member, OsGH3-2, modulates auxin and abscisic acid levels and differentially affects drought and cold tolerance in rice. Journal of experimental botany. 2012; 63(18): 6467-6480.

12. Junichi Ueda, Kensuke Miyamoto, Miyako Aoki. Jasmonic Acid Inhibits the IAA-Induced Elongation of Oat Coleoptile Segments: a Possible Mechanism Involving the Metabolism of Cell Wall Polysaccharides. Plant and Cell Physiology. 1994; 35(7): 1065-1070.

13. Yoshiko Koshitaa, ToshioTakaharaa, Tatsushi Ogataa. Involvement of endogenous plant hormones (IAA, ABA, GAs) in leaves and flower bud formation of satsuma mandarin (Citrus unshiu Marc.). Scientia Horticulturae, 1999; 79 (3-4): 185-194.

14. Yongyin Wang, Susan, MopperKarl et al. Effects of Salinity on Endogenous Aba, laa, Ja, and Sa in Iris hexagona. Journal of Chemical Ecology. 2001; 27(2):327-342.

15. García-Martín, J.A.ManzaneraEmail, M.E.González-Benito.Effect of exogenous ABA on embryo maturation and quantification of endogenous levels of $A B A$ and IAA in Quercus suber somatic embryos. Plant Cell, Tissue and Organ Culture. 2005; 80(2): 171-177.

16. Kazan K., Manners J.M. Linking development to defense: auxin in plant pathogen interactions. Trends Plant Sci. 2009; 14:1360-1385.

17. Brian Jones, Sara Andersson Gunnera, Sara V Petersson. Cytokinin Regulation of Auxin Synthesis in Arabidopsis Involves a Homeostatic Feedback Loop Regulated via Auxin and Cytokinin Signal Transduction.The Plant Cell. 2010; 22: 2956-2969.

18. Li Mengsha, Yan Xiufeng. Jasmonic acid signaling in plants and its biological functions in relation to environment. Acta Ecologica Sinica. 2014; 34 (23): 6779-6788.

19. Kim J, Harter K, Theologis A. Protein-protein interactions among the Aux/IAA proteins. Proceedings of the National Academy of Sciences. 1997; 94(22): 11786-11791.

20. Ulmasov T, Murfett J, Hagen G. Aux/IAA proteins repress expression of reporter genes containing natural and highly active synthetic auxin response elements. The Plant Cell. 1997; 9(11): 1963-1971.

21. Dharmasiri N, Dharmasiri S, Estelle M. The F-box protein TIR1 is an auxin receptor. Nature. 2005; 435 : 441-445.

22. DosSantos Maraschin F, MemelinkJ, OffringaR. Auxin-induced, SCFTIR1-mediated polyubiquitination marks AUX/IAA proteins for degradation. The Plant Journal. 2009; 59(1): 100-109.

23. Vanneste S, Friml J. Auxin: a trigger for change in plant development. Cell. 2009; 136(6): 1005-1016.

24. Maheshwari, S. C., S. Guha, and S. Gupta. Effect of indoleacetic acid on the incorporation of orthophosphate and adenine into plant nuclei in vitro. Biochim. Biophys. Acta. 1966; 117: 470-472.

25. Cherry J. H. Nucleic acid biosynthesis in seed germination: influences of auxin and growth regulating substances. Ann. N.Y. Acad. Sci. 1967; 144: 154-168.

26. Allison C. Mallory, David P. Bartel. MicroRNA-Directed Regulation of Arabidopsis auxin repsonse factor17 Is Essential for Proper Development and Modulates Expression of Early Auxin Response Genes. The plant cell. 2005; 17 (5): 1360-1375. 
27. Eleonora Cominelli, Galbiati M, Vavasseur A. A guard-cell-specific MYB transcription factor regulates stomatal movements and plant drought tolerance. Current biology. 2005; 15(13): 1196-1200.

28. Yong Xiang, Ning Tang, Hao Du. Characterization of OsbZIP23 as a Key Player of the Basic Leucine Zipper Transcription Factor Family for Conferring Abscisic Acid Sensitivity and Salinity and Drought Tolerance in Rice. Plant Physiology. 2008; 138: 1938-1952.

29. Christoph Weiste, Wolfgang Dröge-Laser. The Arabidopsis transcription factor bZIP11 activates auxin-mediated transcription by recruiting the histone acetylation machinery. Nature communications. 2014; 5: 1-12.

30. Zhao Gui-Qin, Wang Suo-Min, Ren Ji-Zhou. Research progress on genetic transformation and ecological adaptability in white clover. Acta Ecologica Sinica. 2004; 24(3): 592-598.

31. Barrs H D, Weatherley P E. A re-examination of the relative turgidity techniques for estimating water deficits in leaves. Australian Journal of Biological Sciences. 1962; 15:413-428.

32. Qi Zhou. Experimental guidance of Plant Physiology [M]. Beijing: China Agriculture Press. 2000:161174.

33. Xiangqing Pan, Ruth Welti, Xuemin Wang. Quantitative analysis of major plant hormones in crude plant extracts by high-performance liquid chromatography-mass spectrometry. Nature Protocols. 2010; 5 (6):985-991.

34. Xia $X$ J, Wang $Y$ J, Zhou $Y$ H. Reactive oxygen species are involved in brassinosteroid-induced stress tolerance in cucumber. Plant Physiology. 2009; 150(2): 801-814.

35. Haitao Shi, Li Chen, TiantianYe, Xiaodong Liu. Modulation of auxin content in Arabidopsis confers improved drought stress resistance. Plant Physiology and Biochemistry. 2014; 82: 209-217.

36. Yasin Ashraf, Nazila Azhar, M Hussain. HussainIndole acetic acid (IAA) induced changes in growth, relative water contents and gas exchange attributes of barley (Hordeum vulgare L.) grown under water stress conditions. Plant Growth Regul. 2006; 50:85-90.

37. Yamaguchi-Shinozaki K, Shinozaki K. Transcriptional regulatory networks in cellular responses and toletance to dehydrationand cold stresses. Annual Review of Plant Biology. 2006; 57:781-803.

38. Uno Y, Furihata T, Abe H, Yoshida R, Shinozaki K, Yamaguchi-Shinozaki K. Arabidopsis basic leucine zipper transcription factors involved in an abscisic acid-dependent signal transduction pathway under drought and high-salinity conditions. Proc Natl Acad Sci USA. 2000; 97:11632-11637.

39. Popko J, Hänsch R, Mendel R R. The role of abscisic acid and auxin in the response of poplar to abiotic stress. Plant Biology. 2010;12(2): 242-258.

40. Xu W, Jia L, Shi W. Abscisic acid accumulation modulates auxin transport in the root tip to enhance proton secretion for maintaining root growth under moderate water stress. New Phytologist. 2013; 197(1): 139-150.

41. Saini S, Sharma I, Kaur N. Auxin: a master regulator in plant root development. Plant cell reports. 2013; 32(6): 741-757. 
42. Teng K, Li J, L iu L. Exogenous $A B A$ induces drought tolerance in upland rice: the role of chloroplast and ABA biosynthesis-related gene expression on photosystem II during PEG stress. Acta physiologiae plantarum. 2014; 36(8): 2219-2227.

43. John J. Ross, Damian P. O’Neill, Jennifer J. Smith. Evidence that auxin promotes gibberellin A1 biosynthesis in pea. The plant journal. 2000; 21(6):547-552.

44. John Ross, Damian O'Neill (). New interactions between classical plant hormones. TRENDS in Plant Science. 2001; 6(1):1-3.

45. Saibo N J, Vriezen W H, Beemster G T, van Der Straeten D (). Growth and stomata development of Arabidopsis hypocotyls are controlled by gibberellins and modulated by ethylene and auxins. Plant $\mathrm{J}$. 2003; 33 (6):989-1000.

46. Merewitz E B, Du H, Yu W. Elevated cytokinin content in ipt transgenic creeping bentgrass promotes drought tolerance through regulating metabolite accumulation. Journal of Experimental Botany. 2012; 63 (3): 1315-1328.

47. Nordström A, Tarkowski P, Tarkowska D. Auxin regulation of cytokinin biosynthesis in Arabidopsis thaliana: a factor of potential importance for auxin-cytokinin-regulated development. Proceedings of the National Academy of Sciences of the United States of America. 2004; 101(21): 8039-8044.

48. Moriwaki T, Miyazawa Y, Kobayashi A. Hormonal regulation of lateral root development in Arabidopsis modulated by MIZ1 and requirement of GNOM activity for MIZ1 function. Plant Physiology. 2011; 157(3): 1209-1220.

49. Jones B, Gunnerås S A, Petersson S V. Cytokinin regulation of auxin synthesis in Arabidopsis involves a homeostatic feedback loop regulated via auxin and cytokinin signal transduction. The Plant Cell. 2010; 22(9): 2956-2969.

50. Fernández-Arbaizar A, Regalado J J, Lorenzo O. Isolation and characterization of novel mutant loci suppressing the ABA hypersensitivity of the Arabidopsis coronatine insensitive 1-16 (coi1-16) mutant during germination and seedling growth. Plant and Cell Physiology. 2012; 53(1): 53-63.

51. Ollas C, Arbona V, GóMez-Cadenas A. Jasmonoyl isoleucine accumulation is needed for abscisic acid build-up in roots of Arabidopsis under water stress conditions. Plant, cell \& environment. 2015; 38(10): 2157-2170.

52. Hayat S, Hasan S A, Fariduddin Q. Growth of tomato (Lycopersicon esculentum) in response to salicylic acid under water stress. Journal of Plant Interactions. 2008; 3 (4): 297-304.

53. Alonso-Ramírez A, Rodríguez D, Reyes D. Evidence for a role of gibberellins in salicylic acidmodulated early plant responses to abiotic stress in Arabidopsis seeds. Plant Physiol. 2009; 150(3):1335-1344.

54. Cheol Park H, Cha J Y, Yun D J. Roles of YUCCAs in auxin biosynthesis and drought stress responses in plant. Plant signaling \& behavior. 2013; 8(6): 337-349.

55. Wang S K, Bai Y H, Shen C J. Auxin-related gene families in abiotic stress response in Sorghum bicolor. Functional \& integrative genomics. 2010; 10(4): 533-546. 
56. Andrew T., Groover, Amy Pattishall. IAA8 expression during vascular cell differentiation. Plant Molecular Biology. 2003; 51(3):427-435.

57. Carole Bassa, Isabelle Mila, Mondher Bouzayen. Phenotypes Associated with Down-Regulation of SIIAA27 Support Functional Diversity Among Aux/IAA Family Members in Tomato. Plant and Cell Physiology. 2012; 53(9): 1583-1595.

58. Dekai Wang, Kemei Pei,Yaping Fu. Genome-wide analysis of the auxin response factors (ARF) gene family in rice (Oryza sativa).Gene. 2007; 394 (1-2):13-24.

59. Foyer $\mathrm{C} \mathrm{H}$, Descourvieres P, Kunert K J. Protection against oxygen radicals: an important defence mechanism studied in transgenic plants. Plant, Cell \& Environment. 1994; 17(5): 507-523.

60. Shenwei Zhang, Chen Hui Li, Cao J. Altered architecture and enhanced drought tolerance in rice via the down-regulation of indole-3-acetic acid by TLD1/OsGH3. 13 activation. Plant physiology. 2009; 151(4): 1889-1901.

61. Khan S, Stone J M. Arabidopsis thaliana GH3.9 influences primary root growth. Planta. 2007; 226(1): 21-34.

62. Golldack D, Lüking I, Yang O. Plant tolerance to drought and salinity: stress regulating transcription factors and their functional significance in the cellular transcriptional network. Plant cell reports, 2011; 30(8): 1383-1391.

63. Xiang Y, Tang N, Du H. Characterization of OsbZIP23 as a key player of the basic leucine zipper transcription factor family for conferring abscisic acid sensitivity and salinity and drought tolerance in rice. Plant physiology. 2008; 148(4): 1938-1952.

64. Guoqiang Fan, Limin Wang, Minjie Deng. Changes in Transcript Related to Osmosis and Intracellular Ion Homeostasis in Paulownia tomentosa under Salt Stress. Front Plant Sci. 2016; 7: 384.

65. Todaka D, Shinozaki K, Yamaguchi-Shinozaki K. Recent advances in the dissection of drought-stress regulatory networks and strategies for development of drought-tolerant transgenic rice plants. Frontiers in plant science. 2015; 6: 84 .

66. Liu Q, Kasuga M, Sakuma Y. Two transcription factors, DREB1 and DREB2, with an EREBP/AP2 DNA binding domain separate two cellular signal transduction pathways in drought-and low-temperatureresponsive gene expression, respectively, in Arabidopsis. The Plant Cell. 1998; 10(8): 1391-1406.

67. Xiong H, Li J, Liu P. Overexpression of OsMYB48-1, a novel MYB-related transcription factor, enhances drought and salinity tolerance in rice. PLoS One. 2014; 9(3): e92913.

68. Eulgem T, Somssich I E. Networks of WRKY transcription factors in defense signaling. Current opinion in plant biology. 2007; 10(4): 366-371.

69. Tripathi P, Rabara R C, Rushton P J. A systems biology perspective on the role of WRKY transcription factors in drought responses in plants. Planta. 2014; 239(2): 255-266.

70. Ren X, Chen Z, Liu Y. ABO3, a WRKY transcription factor, mediates plant responses to abscisic acid and drought tolerance in Arabidopsis. The Plant Journal. 2010; 63(3): 417-429. 
71. Almazroue H A. Identification, Cloning, and Expression of Tobacco Responsive to Dehydration like Protein (RD22), SBIP-355 and Its Role in SABP2 Mediated SA Pathway in Plant Defense. 2014.

72. Shinozaki K, Yamaguchi-Shinozaki K. Molecular responses to drought stress [M]. Amsterdam, Elsevier Science BV. 1998.

73. Tomohiro Kiyosue, KazukoYamaguchi-Shinozaki, Kazuo Shinozaki. Characterization of two cDNAs (ERD11 and ERD13) for dehydration-inducible genes that encode putative glutathione S-transferases in Arabidopsis thaliana L. Federation of European Biochemical Societies. 1993; 335(2): 189-192.

74. Abe H, Yamaguchi-Shinozaki K, Urao T. Role of Arabidopsis MYC and MYB homologs in drought-and abscisic acid-regulated gene expression. The Plant Cell. 1997; 9(10): 1859-1868.

75. Yuehui He, Susheng Gan. A Gene Encoding an Acyl Hydrolase Is Involved in Leaf Senescence in Arabidopsis. The plant cell. 2002; 14(4):805-815.

76. Noh Y S, Amasino R M. Identification of a promoter region responsible for the senescence-specific expression of SAG12. Plant molecular biology. 1999; 41(2): 181-194.

77. Im Kim J, Murphy A S, Baek D. YUCCA6 over-expression demonstrates auxin function in delaying leaf senescence in Arabidopsis thaliana. Journal of experimental botany. 2011; 62(11): 3981-3992.

\section{Tables}

Table 1 Primer sequences of the genes related to IAA and their corresponding GeneBank accession numbers

\begin{tabular}{llll}
\hline Target gene & Accession No. & \multicolumn{1}{c}{ Forward primer (5'-3') } & \multicolumn{1}{c}{ Reverse primer (5'-3') } \\
\hline GH3.1 & MF099746 & TCGTCAACTTCTATACAGCCTTCT & CACTTGGTGTCCTTGTTTCTGA \\
GH3.3 & MF099747 & TGACTCGGACAAAACAGACG & CTTCATCACTAGGTGGATTAGAAG \\
\hline GH3.5 & MF099748 & GATGCTGAGAATGTTCAAAAGG & AGAAACATCACCACCAACCA \\
\hline GH3.6 & MF099749 & GAAGAAGAGTTAGGGAGGAGAAG & CCAGGTGTTTTAGCCTCAGAT \\
\hline GH3.9 & MF099750 & CATTGAAGCAGTGGTTACAGG & CACCAAAGTAACACTCAGAAGAAG \\
\hline IAA8 & MF099751 & ATGCTATCGCCTAGACCTGTT & TGCCTTAGATGCTGGCTGTG \\
\hline IAA27 & MF099752 & CCTCAAAGCTACTGAACTGAGAC & ACCCATTTACCAGAACCTCC \\
\hline ARF & MF099753 & TCTGCTGAGTTTACGAGGGTTC & GGTTTTGTTGCTTGCTGATGC \\
\hline GAPDH & F968420.1 & TTACAGAAAGGCACAGGGATGAC & CGGGAGACTAAGGAGGAACTAT \\
\hline
\end{tabular}

Table 2 Primer sequences of drought-induced transcription factors and drought-induced genes and their corresponding GeneBank accession numbers 


\begin{tabular}{llll}
\hline Target gene & Accession No. & \multicolumn{1}{c}{ Forward primer (5'-3') } & Reverse primer (5'-3') \\
\hline DREB2 & EU846194.1 & CAAGAACAAGATGATGATGGTGAAC & AAGAAGAAGAATTGGAGGAGTCATG \\
DREB3 & EU846196.1 & GCTCAATAGGACTCAACCAACTCAC & TGACGTTGTCTAACTCCACGGTAA \\
\hline DREB4 & EU846198.1 & CTTGGTTGTGGAGATAATGGAGC & AAGTTGCAATCTGAATTCTGAGGAC \\
\hline DREB5 & EU846200.1 & GCGATAGGTTCAAAGAAAGGGTG & AGAGCAGCATCTTGAGCAGTAGG \\
\hline bZIP11 & MF099755 & TTCCTTGCCTCCACTTAGTCC & GATCGTCTGTGCCCTTTACG \\
\hline bZIP 37 & MF099754 & GAACCCGTCTGAACATAACTGAA & AGCGACTTTGGAGCCATCAT \\
\hline bZIP 107 & MF099756 & AGACCCACCAATAACCAAACTG & CATAAAAGGAAGAAGAAGGAGGAG \\
\hline MYB14 & JN117923.1 & GACGAAGAGAAAGAACTATCCGCA & TTGATCCGAACAAGGCGACA \\
\hline MYB48 & MF099757 & CGAGAAAGGTCATACAAACAAAGG & TGAGGTCAGGGCGGAGATAG \\
\hline MYB112 & MF099758 & GCCAGGAAGAACCGACAATG & GCCAGGAAGAACCGACAATG \\
\hline WRKY2 & MF099759 & GGCACATAACCACCCGAAAC & AAATTAGCCCAGCCACGATC \\
\hline WRKY56 & MF099760 & GCTCTTTTGCTCCAAGCTGTC & AATTGAGGCTCACGCTACGG \\
\hline WRKY108715 & MF099761 & GAACAGACCAACTCCAAACAGC & GCAAATCAGGATGGAAAGGAC \\
\hline ERF019 & MF099762 & GATATTGCTATGGATGTCGATGC & AAGTCCTCTTGTTGGCTAGAAACT \\
\hline ERF098 & MF099763 & TGCGGCGGAGATACGAGAT & GGAAGAAGTGGGCTTAGAAGGA \\
\hline ERF110 & MF099764 & TTCGCCATCGCTTTCTTTGT & TCCGCTACGAGATTGATCTTCC \\
\hline ERD & XM_003612152.2 & CCATCGCTGTCTATGCTCGTA & TTCTTCCTCGTCTGAATCGGTA \\
\hline RD22 & XM_003588503.2 & GTCCAAACTTCCCACAACTCA & CCTCCTTTTCCTACAGCTACTG \\
\hline GAPDH & JF968420.1 & TTACAGAAAGGCACAGGGATGAC & CGGGAGACTAAGGAGGAACTAT \\
\hline SAG101 & XM_004489275.2 & CATTTCGTACTCGCTGGCTCT & CACGTAATCCTTACCACCGTCT \\
\hline SAG102 & XM_003590568.2 & ATCATTGGACTTGGTCTTGTTGG & GAAGTGGGCAAGGGAGGAAT \\
\hline
\end{tabular}

\section{Figures}



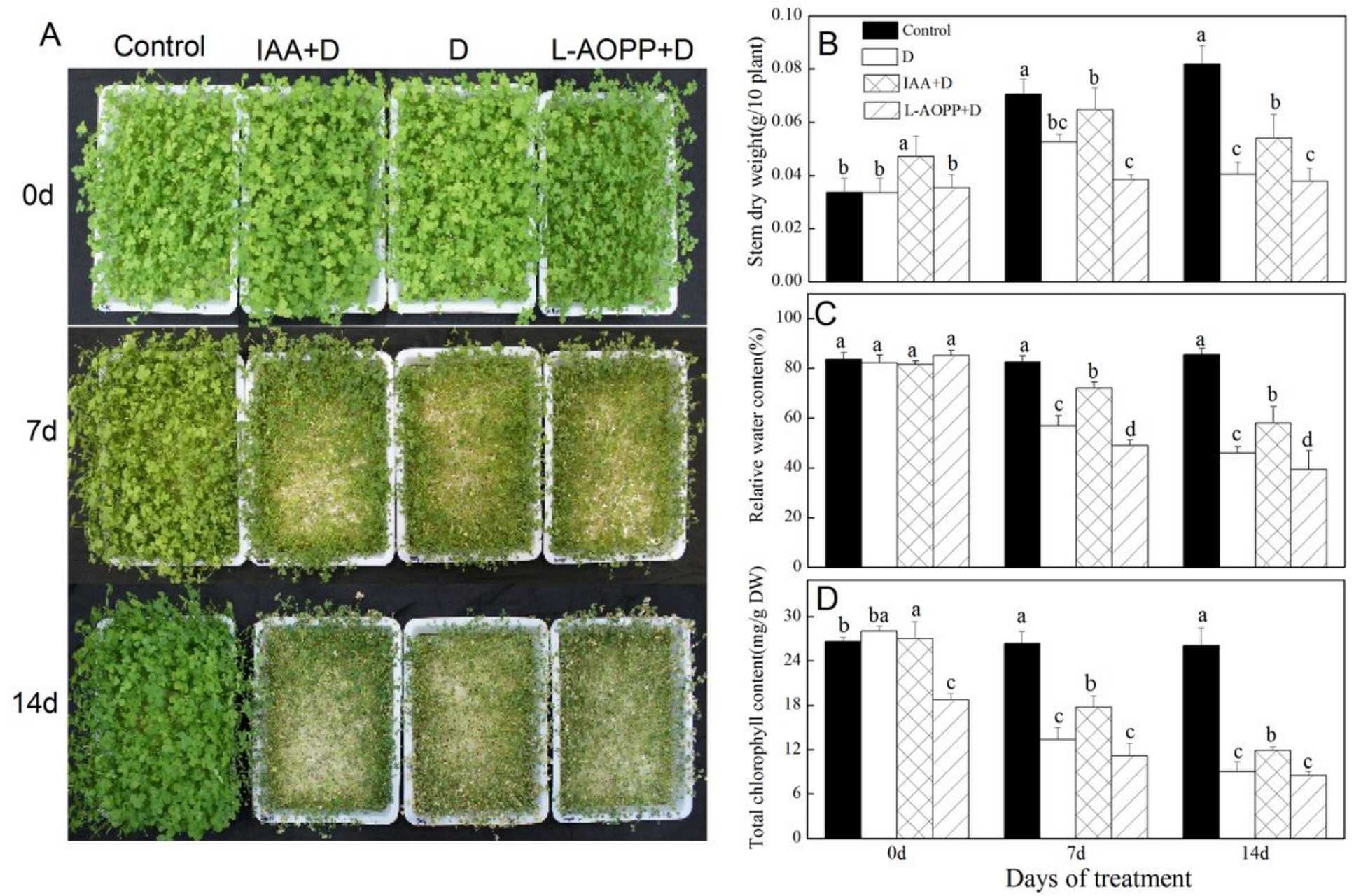

Figure 1

IAA /L-AOPP pretreatment, drought stress and sampling time. Figure 1. Morphological appearance(A), Stem dry weight (B), relative water content (C) and total chlorophyll content (D) of white clover in different treatments. Vertical columns represent Mean+STD $(n=4)$. The same letter indicates no significant difference and the different letters indicate significant difference in a particular day of treatment $(\mathrm{P}<$ 0.05). 

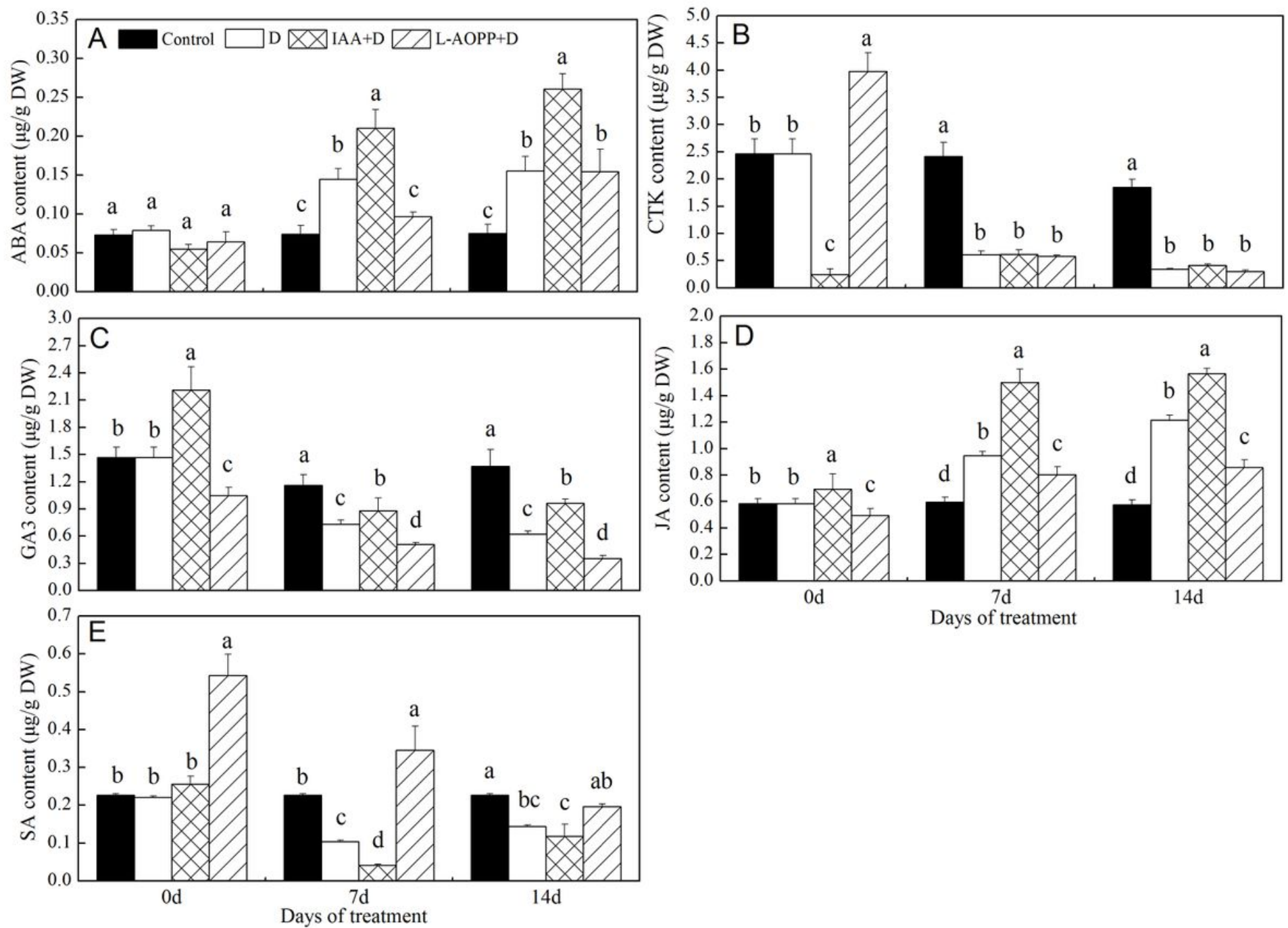

Figure 2

Endogenous IAA content $(A)$ and relative Expression of auxin response genes of white clover leaves in different treatments. ARF (B). GH3.1 (C), GH3.3 (D), GH3.5 (E), GH3.6 (F), GH3.9 (G), IAA8 (H), IAA27 (J) and Vertical columns represent Mean+STD $(n=4)$. The same letter indicates no significant difference and the differenet letters indicate significant difference in a particular day of treatment $(P<0.05)$. 

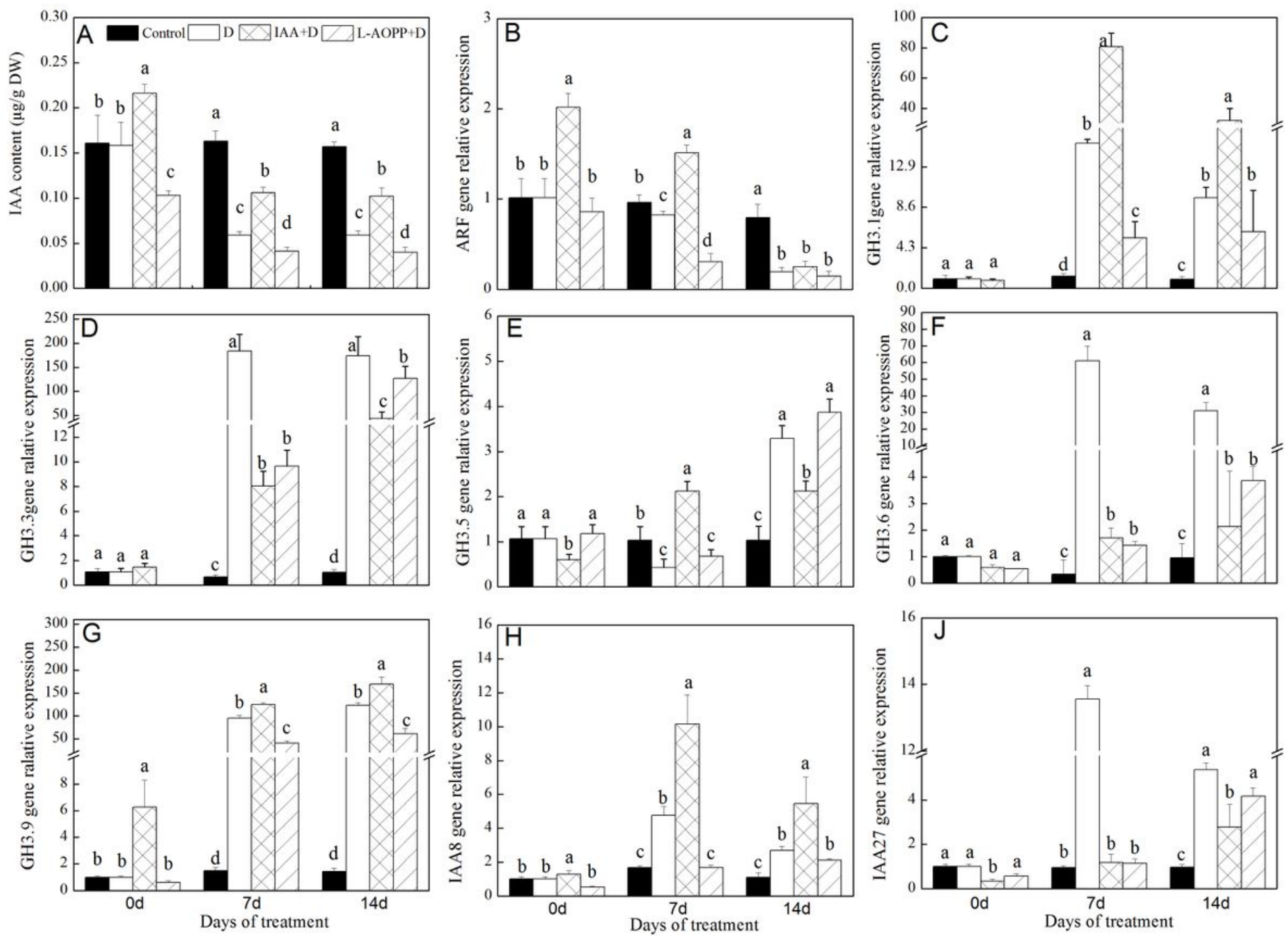

Figure 3

Contents of other major phytohormones of white clover leaves in different treatments. ABA content $(A)$, CTK content (B), GA content (C), JA content (D) and SA content (E).Vertical columns represent Mean+/std $(n=4)$. The same letter indicates no significant difference and the different letters indicate significant difference in a particular day of treatment. $(P<0.05)$. 

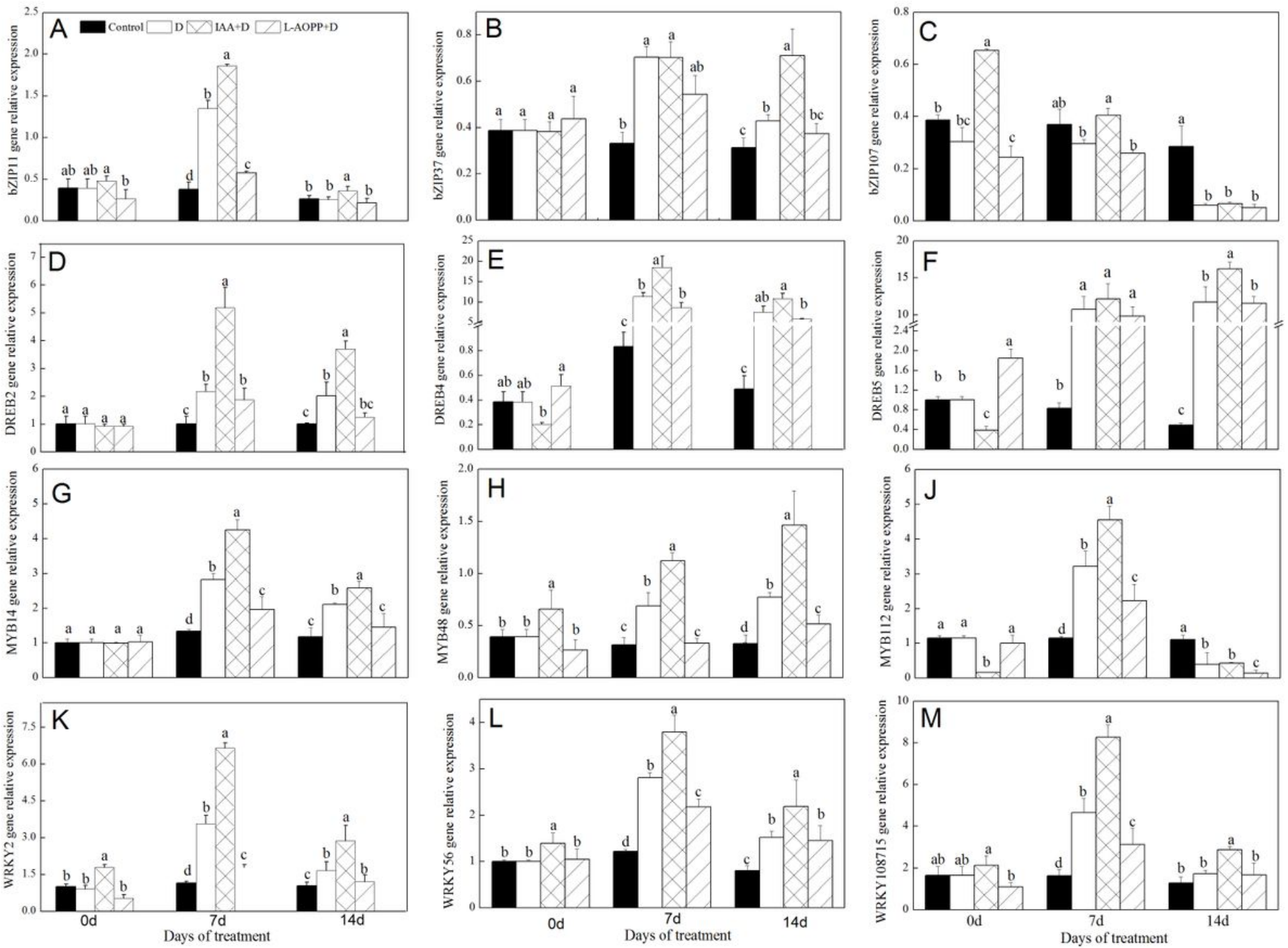

Figure 4

Expression of drought-induced transcriptional factors of white clover leaves in different treatments. bZIP11 (A), bZIP 37 (B), bZIP 107 (C), DREB2 (D), DREB4 (E), DREB5 (F), MYB14 (G), MYB48 (H), MYB112 (J),WRKY2 (K), WRKY56 (L) and WRKY108715 (M).Vertical columns represent Mean+STD ( $n=4)$. The same letter indicates no significant difference and the different letters indicate significant difference under a particular day of treatment $(P<0.05)$. 

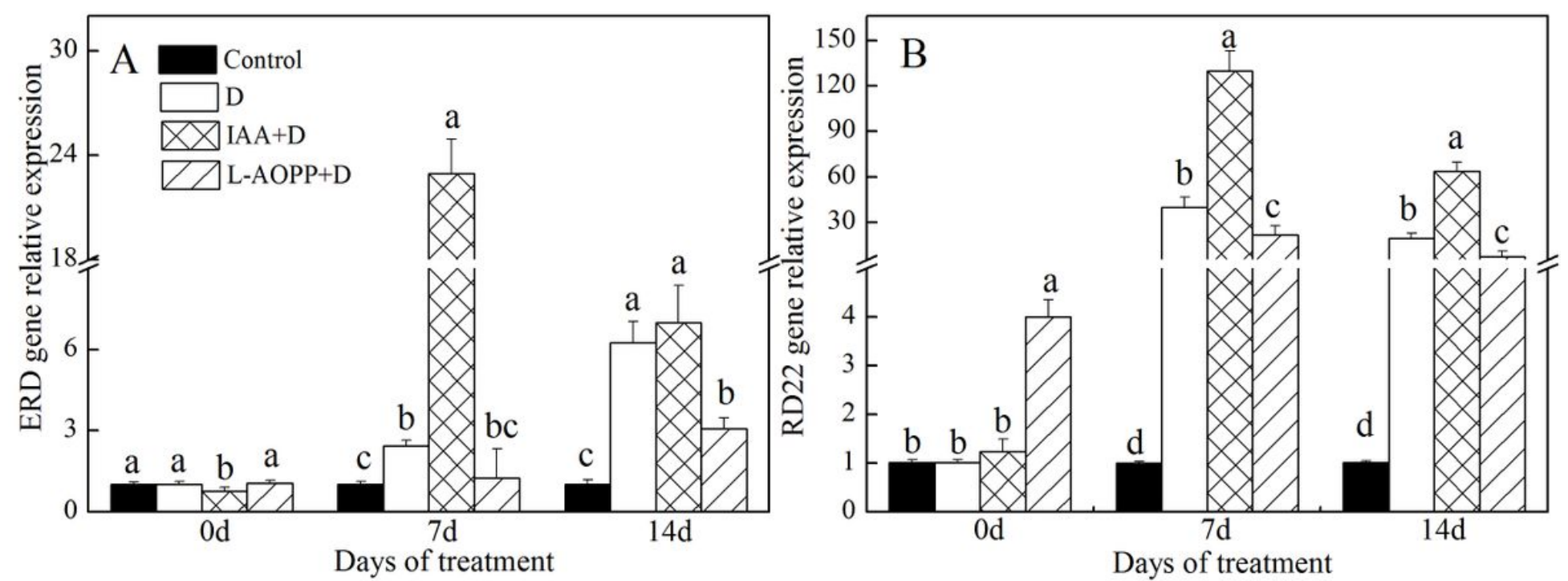

Figure 5

Expression of drought-induced genes of white clover leaves in different treatments. ERD (A) and RD22 (B). Vertical columns represent Mean+STD $(n=4)$. The same letter indicates no significant difference and the different letters indicate significant difference in a particular day of treatment $(P<0.05)$.
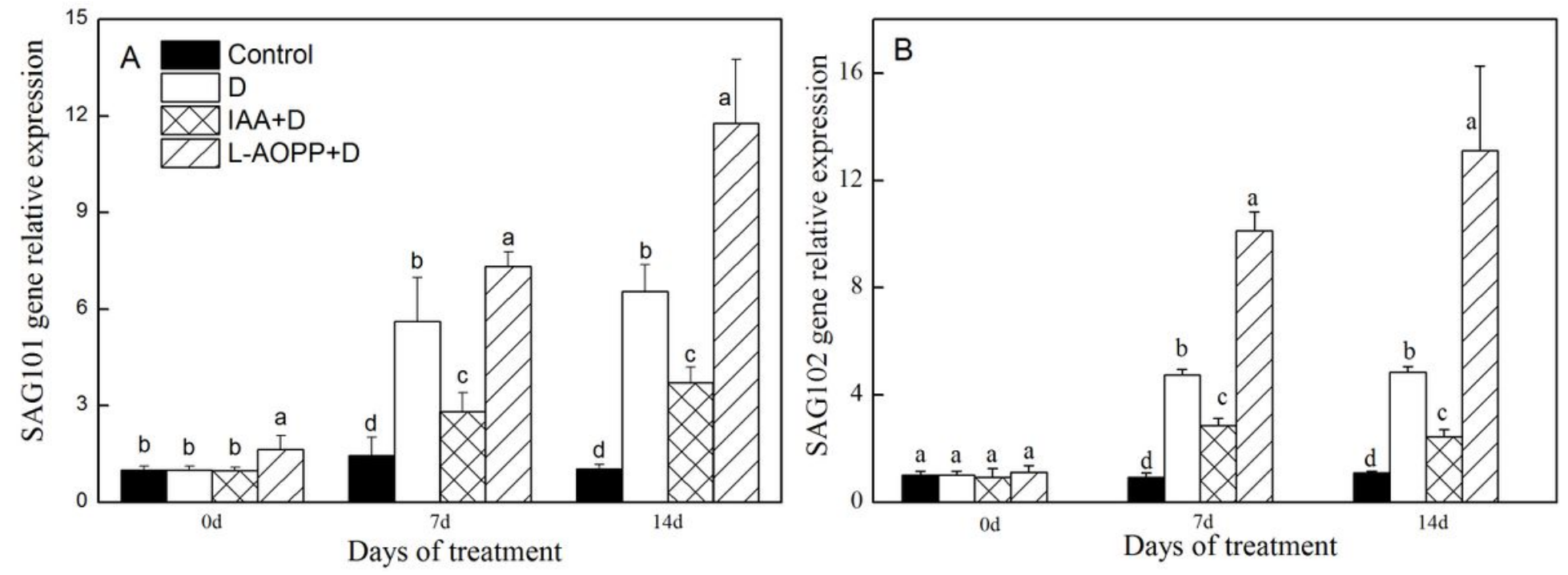

Figure 6

Expression of senescence-associated genes of white clover leaves in different treatments. SAG101 (A) and SAG102 (B). Vertical columns represent Mean+STD $(n=4)$. The same letter indicates no significant difference and the different letters indicate significant difference in a particular day of treatment $(\mathrm{P}<$ 0.05). 


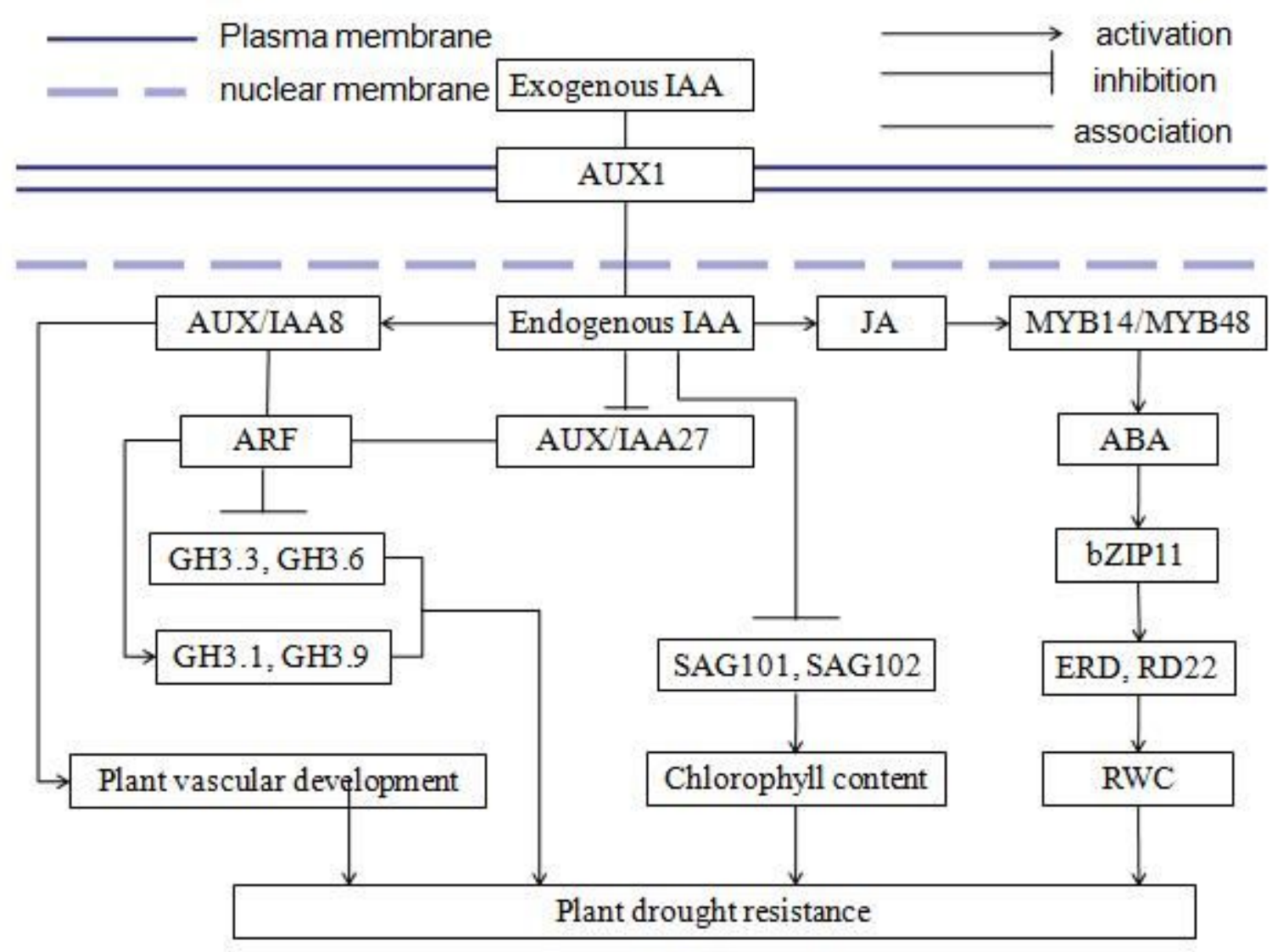

\section{Figure 7}

Hypothesis working model for IAA's improving droght resistance in white colver.

\section{Supplementary Files}

This is a list of supplementary files associated with this preprint. Click to download.

- keymessage.doc 